\title{
Trisomy 10qter confirmed by in situ hybridisation
}

\author{
V Briscioli, G Floridia, E Rossi, A Selicorni, F Lalatta, O Zuffardi
}

\begin{abstract}
We report a boy with multiple congenital anomalies compatible with trisomy for the distal region of the long arm of chromosome 10 and a male karyotype with one $18 p+$. In situ hybridisation with a cDNA for ornithine aminotransferase (OAT), whose locus maps to 10q26, confirmed the clinical suspicion of distal trisomy 10q. Subterminal localisation of the labelling signals on chromosome 10 and on the der(18) indicated the localisation of the OAT locus in the proximal part of 10q26. Two clusters of labelling signals were also found on the pericentromeric and proximal portion of the $X$ chromosome short arm, thus confirming the presence in this region of two non-adjacent OAT pseudogenes. The phenotypic similarities of this patient to previously reported cases provide further support for the delineation of trisomy 10qter as a specific, clinically recognisable syndrome.

(f Med Genet 1993;30:601-3)
\end{abstract}

De novo unbalanced structural anomalies have a frequency of $0.038 \%,{ }^{1}$ most of them being unbalanced translocations. When detected in liveborns, these imbalances can pose problems in karyotype interpretation since the chromosome region transposed to the recipient chromosome is usually short enough to allow viability of the zygote and thus it can be difficult to identify it by banding patterns. We present a boy with multiple congenital anomalies, in whom chromosome analysis showed a de novo $18 p+$ male karyotype. Clinical characterisa-

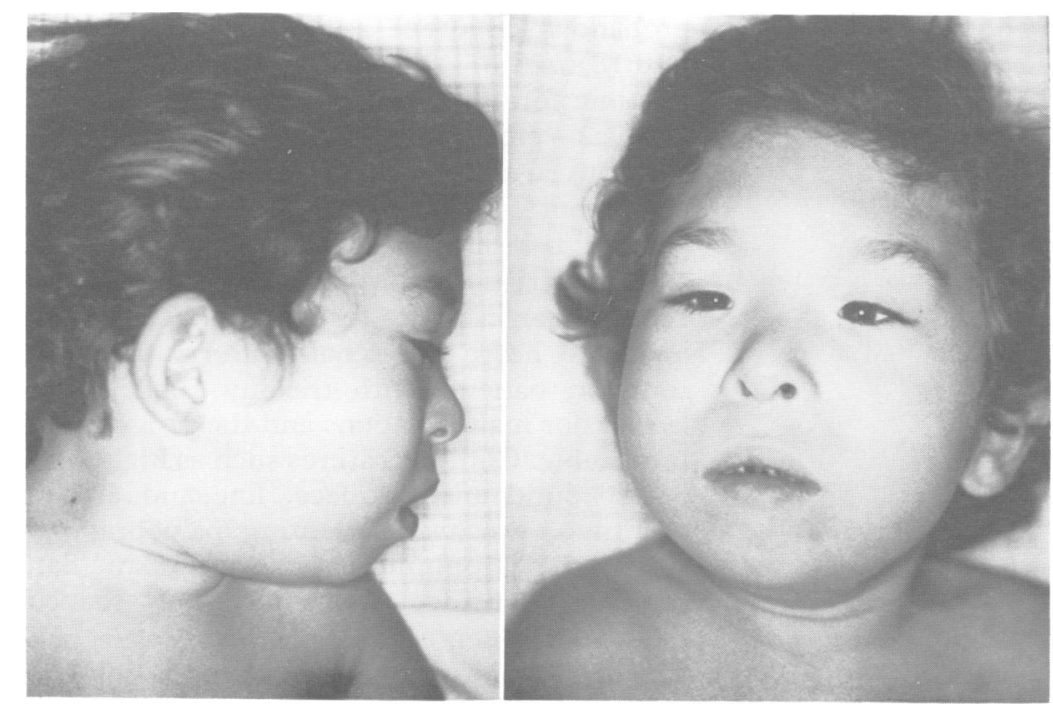

Figure 1 The patient at 6 years. tion of the patient when 6 years old led to the suspicion of trisomy for the distal region of $10 \mathrm{q}$. In situ hybridisation with a probe for the region confirmed the clinical diagnosis.

\section{Case report}

The proband was the first child of healthy, unrelated parents; the mother was aged 29 years and the father 34 years. The mother had previously had two spontaneous abortions. The proband was born by normal delivery after an uneventful pregnancy at 41 weeks. Birth weight was $3250 \mathrm{~g}$ (50th centile), length $48 \mathrm{~cm}$ (50th centile), and head circumference $34 \mathrm{~cm}$ (50th centile). At birth he had respiratory distress and severe hypotonia. At 7 months of age he was evaluated because of poor feeding, psychomotor retardation, and craniofacial dysmorphism. Cardiological and ophthalmological examination was normal, but skeletal $x$ ray showed marked thoracolumbar kyphoscoliosis and a dysplastic hip. Clinical examination at the age of 6 years showed height and weight below the 3rd centile, head circumference on the 50th centile, inner canthal distance on the 97th centile, outer canthal distance on the 90th centile, total hand and foot length below the 3rd centile, and middle finger length on the 50th centile. The face (fig 1) was round with a high and large forehead and bitemporal constriction. The eyebrows were fine and arched, the palpebral fissures were downward slanting with blepharophimosis, the nose was short with a flat nasal bridge, the mouth was bow shaped, the ears were low set, and the neck was short. Cleft palate, severe kyphoscoliosis, and coxa valga were present. The fingers were long and thin with camptodactyly. He had moderate mental retardation (psychological tests showed an IQ of 63).

\section{CHROMOSOME ANALYSIS}

Chromosome analysis was performed on trypsin $G$ banded preparations from lymphocyte cultures. The proband had a male karyotype with extra material on the short arm of a chromosome 18, which appeared slightly larger than a chromosome 16 . No cell line is available from this patient. Parental karyotypes were normal. Q, R, DA-DAPI and high resolution banding ${ }^{2}$ were performed but no unequivocal interpretation could be made although it was clear that the extra material was entirely euchromatic. Since the malformations were consistent with a partial duplication of $10 \mathrm{q}$, we decided to perform in situ hybridisation with a probe mapping to the distal region of $10 \mathrm{q}$. 
Results of in situ hybridisation of hu OAT 6 to the proband's metaphases*.

No of mitoses with

at least one FISH

signal at 10q26, Xp11,

or der(18)distal $\mathbf{p} /$

FISH signals at

\begin{tabular}{lccc}
\cline { 2 - 4 } total no of mitoses & $10 \mathrm{q} 26$ & $\operatorname{der}(18)$ distal $\mathrm{p}$ & $\mathrm{Xp11}$ \\
\hline $50 / 64$ & 43 in 32 mitoses & 20 in 17 mitoses & 42 in 27 mitoses \\
\hline
\end{tabular}

* Only mitoses with one to eight FISH signals have been considered.

IN SITU HYBRIDISATION

In situ hybridisation was performed with a probe for the ornithine- $\delta$-aminotransferase (OAT) gene whose structural locus maps to $10 \mathrm{q} 26$ though OAT related sequences are also present on $\mathrm{Xp}^{34}$ The human cDNA hu OAT $6^{5}$ in pGEM-4 vector was biotin-16-dUTP labelled by nick translation according to the Boehringer-Mannheim protocol. The probe (final concentration $6 \mathrm{ng} / \mu \mathrm{l}$ ) was hybridised in $50 \%$ formamide in $2 \times \mathrm{SSC}$ at $37^{\circ} \mathrm{C}$. Posthybridisation washes were done in $50 \%$ formamide in $2 \times \mathrm{SSC}$ for 20 minutes and in $2 \times \mathrm{SSC}$ for one hour at $42^{\circ} \mathrm{C}$. Detection was done with the ONCOR detection kit with two amplification steps. Chromosomes were counterstained with propidium iodide $(200 \mathrm{ng} / \mathrm{ml}$ in $2 \times \mathrm{SSC}$, five minutes at room temperature) and banded with diamidinophenylindole (DAPI). ${ }^{6}$ After mounting in antifade solution (2\% McIlvaine buffer, pH 7.0, containing $1 \mathrm{mg} / \mathrm{ml}$ of 1,4-phenylendiamin-dihydrochloride in glycerol), slides were evaluated on a Zeiss Axiophot microscope equipped for con-

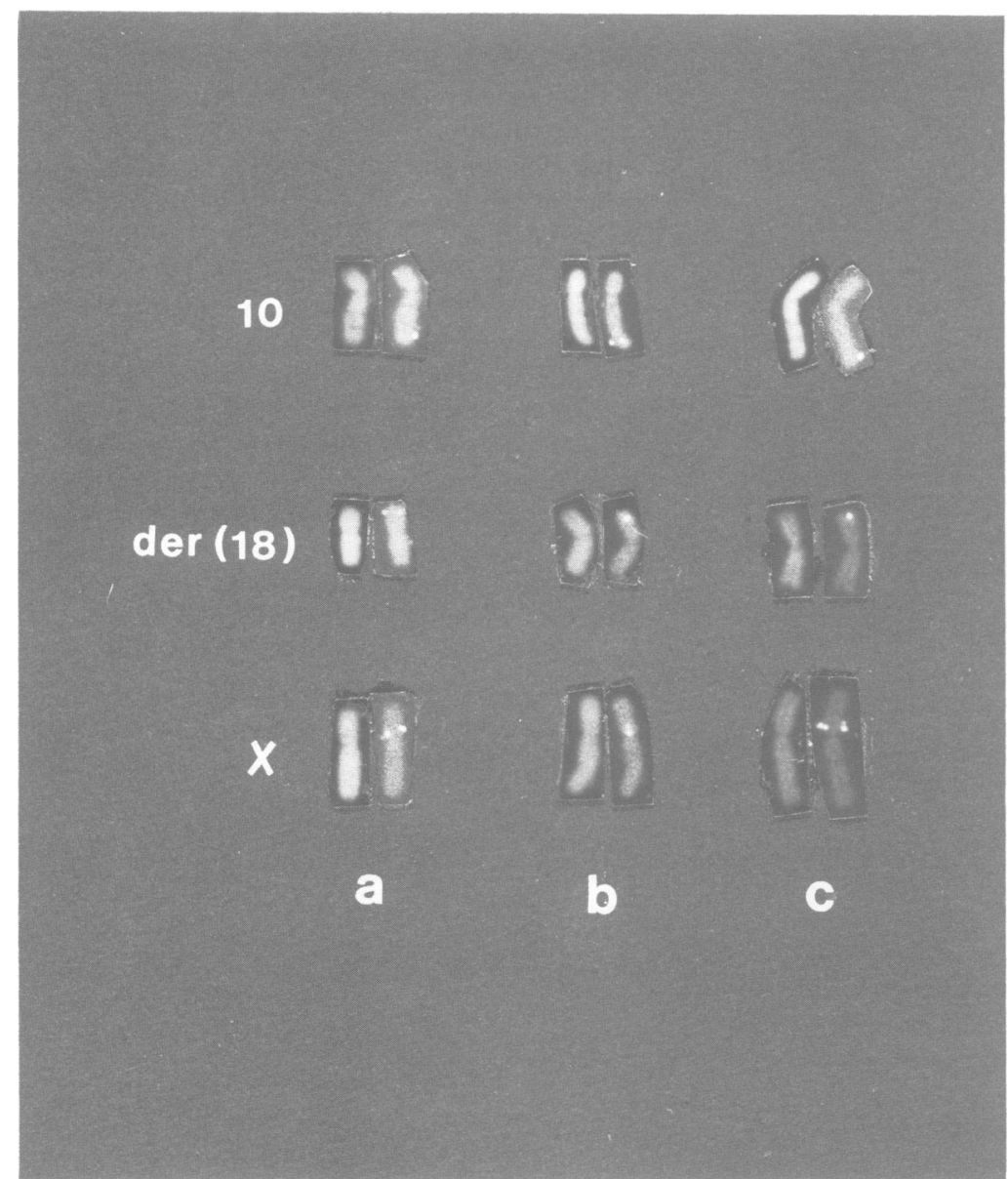

Figure 3 Chromosomes 10, der (18), and $X$ from the proband after in situ hybridisation with hu OAT 6; (left DAPI staining, (right) propidium iodide counterstaining.

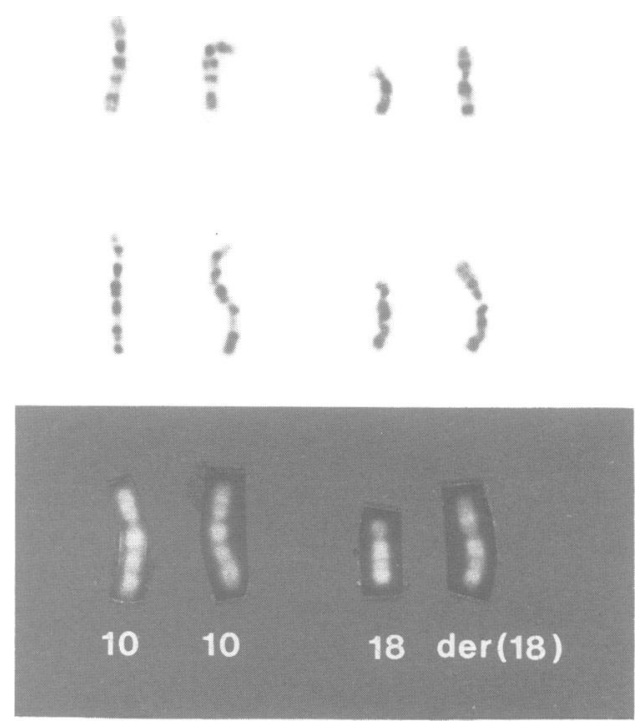

Figure $2 G$ and $Q$ banded chromosomes 10, 18, and $\operatorname{der}(18)$ of the proband.

ventional epifluorescence microscopy (BP 365, FT 395, LP 397 for DAPI staining; BP 450490, FT 510, LP 520 for FITC and propidium iodide).

Results of the in situ hybridisation are given in the table. The distribution of the fluorescent signals shows that the single $\mathrm{X}$ chromosome and the two chromosomes 10 had approximately the same number of hybridised spots and this was roughly double the number of spots found in the $\operatorname{der}(18)$. The localisation of the spots on the chromosome 10 long arm and on the der(18) short arm was subterminal (fig 2). The spots at Xp11, in those mitoses with more than one signal, were alternatively distributed as two asymmetrical signals, one on each chromatid (fig 3a), two signals one over the other (fig $3 \mathrm{~b}$ ), or as two sets of superimposed symmetrical signals (fig $3 \mathrm{c}$ ).

\section{Discussion}

The distribution of hybridisation signals clearly indicates that the extra piece of the $\operatorname{der}(18)$ is constituted by the distal portion of 10q. Together, chromosome banding and hybridisation data led us to interpret the proband's karyotype as $46, \mathrm{XY},-18,+\operatorname{der}(18)$ $\mathrm{t}(10 ; 18)$ (q25;p11.2) (fig 1). Thus the patient is trisomic for $10 \mathrm{q} 25 \rightarrow \mathrm{qter}$ and monosomic for $18 \mathrm{p} 11.2 \rightarrow$ pter.

About 30 cases of distal trisomy for $10 \mathrm{q}$ have been reported, ${ }^{78}$ most of them resulting from a parental translocation. This trisomy results in a characteristic syndrome with more severe clinical manifestations for trisomy $10 \mathrm{q} 24 \rightarrow$ qter owing to heart and renal malformations. In contrast, patients with trisomy $10 \mathrm{q} 25 \rightarrow$ qter lack major malformations and the prognosis is favourable. Clinical features such as high forehead, round and flat face, fine and arched eyebrows, downward slanting palpebral fissures, blepharophimosis, hypertelorism, flat, broad nasal bridge, short nose, bow shaped mouth, low set ears, short neck, cleft palate, and kyphoscoliosis are common to both trisomies. In our case all these features were present together with moderate mental 
retardation, as in most cases with trisomy $10 \mathrm{q} 25 \rightarrow$ qter, and in contrast to cases with trisomy 10q24 $\rightarrow$ qter who have profound mental retardation. All these signs prompted us to suspect trisomy for the distal region of $10 q$ and even after the karyotype delineation we could not detect any clinical sign characteristic of monosomy $18 \mathrm{p}$. The same holds true for a patient reported by Forabosco et al with a karyotype identical to that of our case.

From the cytogenetic point of view, the OAT locus seems to map to the proximal portion of 10q26, as suggested by the subterminal localisation of the fluorescent spots on chromosomes 10 and $\operatorname{der}(18)$. The in situ hybridisation pattern also suggested the presence of two OAT related sequences on Xp. In fact the number of labelling spots on the single $\mathrm{X}$ chromosome was double that found on the $\operatorname{der}(18)$ and similar to that found on the two chromosomes 10. Moreover, two clusters of spots were identified on $\mathrm{Xp}$, one close to the centromere and a second proximal to $\mathrm{Xp} 21$, thus confirming that two OAT related sequences on $\mathrm{Xp}$ map to non-adjacent intervals. ${ }^{4}$
We thank Dr H F Willard for the generous gift of probe hu OAT 6 and Professor M Fraccaro for helpful discussion. This work was supported by Associazione Studio Malformazzioni, Milano.

1 Jacobs PA, Browne C, Gregson N, Joyce C, White $H$. Estimates of the frequency of chromosome abnormalities detectable in unselected newborns using moderate levels of banding. F Med Genet 1992;29:103-8.

2 Dutrillaux B, Viegas-Pequignot E. High resolution R- and G-banding on the same preparation. Hum Genet 1981;57:93-5.

3 Barrett DJ, Bateman B, Sparkes RS, Mohandas T, Klisak I, Inana $\mathbf{G}$. Chromosomal localization of human ornithine aminotransferase gene sequences to $10 \mathrm{q} 26$ and $\mathrm{Xp} 112$. Invest Ophthalmol Vis Sci 1987;28:1037-42.

4 Lafreniere RG, Geraghty MT, Valle D, Shows TB, Willard HF. Ornithine aminotransferase-related sequences map to two nonadjacent intervals on the human $\mathrm{X}$ chromosome two nonadjacent intervals on the hum
short arm. Genomics 1991;10:276-9.

5 Mitchell GA, Looney JE, Brody LC, et al. Human ornithine$\delta$-aminotransferase. CDNA cloning and analysis of the
Mitchel GA, Looney JE, Brody LC, et al. Human ornithe-

6 Schweizer D. Counterstain-enhanced chromosome banding. Hum Genet 1981;57:1-14.

7 Taysi K, Yang V, Monaghan N, Beraha N. Partial trisomy $10 \mathrm{q}$ in three unrelated patients. Ann Genet (Paris) 1983;26:79-85.

8 Schinzel A. Catalogue of unbalanced chromosome aberration in man. Berlin: Walter de Gruyter, 1984:407-9.

9 Forabosco A, Bernasconi S, Giovannelli G, Dutrillaux B. Trisomy of the distal third of the long arm of chromosome 10. Helv Paediatr Acta 1975;30:289-95. 